\title{
Programa Recuperación de Barrios: Una experiencia de urbanismo ciudadano
}

\author{
Alejandra Vio* \\ Claudia Bustos** \\ Daniela Aguirre*** \\ Nelson Morales $* * * *$
}

\section{RESUMEN}

El Programa Recuperación de Barrios del Ministerio de Vivienda y Urbanismo, constituye un esfuerzo de innovación en política social, cuyo objetivo es construir propuestas de desarrollo urbano integral, surgidas del trabajo conjunto de la comunidad, los gobiernos locales y el gobierno central, como expresión de una nueva relación entre el Estado y la ciudadanía en la construcción de ciudad.

Palabras clave: Urbanismo ciudadano - recuperación de barrios - ciudad inclusiva - trabajo comunitario - proyectos colectivos - ciudadanía activa.

\section{Programa recuperação de bairros: uma experiência de urbanismo cidadão}

\section{RESUMO}

O Programa Recuperação de Bairros do Ministério de Moradia e Urbanismo, constitui um esforço de inovação em política social, cujo objectivo é construir propostas de desenvolvimento urbano integral, surgidas do trabalho conjunto da comunidade, os governos locais e o governo central, como expressão de uma nova relação entre o Estado e a cidadania na construção de cidade.

Palavras chave: Urbanismo cidadão - recuperação de bairros - cidade inclusiva - trabalho comunitário - projectos colectivos - cidadania activa

* Chilena, Trabajadora Social, Secretaria Ejecutiva Programa de Recuperación de Barrios, Ministerio de Vivienda y Urbanismo. Correo electrónico: avio@minvu.cl

** Chilena, Socióloga, profesional Programa Recuperación de Barrios, Ministerio de Vivienda y Urbanismo. Correo electrónico: cbustosg@minvu.cl

*** Chilena, Socióloga, profesional Programa Recuperación de Barrios, Ministerio de Vivienda y Urbanismo. Correo electrónico: daguirrel@minvu.cl

**** Chileno, Sociólogo, Doctor en urbanismo, profesional Programa Recuperación de Barrios, Ministerio de Vivienda y Urbanismo. Correo electrónico: nmorales@minvu.cl 


\section{Neighborhood recovery program: an experience of urban citizen}

\section{ABSTRACT}

The Neighborhood Recuperation Program of the Housing and Urbanism Minister of Chile is an innovative effort in public policy. Its objective is to build integral proposals in the urban development field, coming from the joint participation of local community, local government and central government. It is an expression of the new relationship between the State and citizenship in building the city.

Key words: Citizen urbanism - neighborhood recuperation - inclusive city community work - collective projects - active city. 


\section{Antecedentes del programa recuperación de barrios}

El Programa Recuperación de Barrios implementado desde el año 2006 por el Ministerio de Vivienda y Urbanismo, en 200 barrios de todo Chile, es un compromiso de la Presidenta Michelle Bachelet, orientado a mejorar la calidad de vida en barrios con deterioro físico e incorporando a los ciudadanos a ser protagonistas de los cambios de sus entornos.

La recuperación ha sido el resultado del trabajo mancomunado de los vecinos, los gobiernos locales y el gobierno central, sentando las bases de un nuevo urbanismo, que busca construir la ciudad desde los barrios y sus ciudadanos.

Se trata de una recuperación física de los espacios públicos barriales, íntimamente ligada a las condiciones del desarrollo social, comunitario e institucional del territorio. Ello, en busca de ciudades más integradoras, que se construyen barrio a barrio, comunidad a comunidad, con sueños y proyectos colectivos de una ciudad más inclusiva y una ciudadanía más activa.

\section{Contexto urbano del programa recuperación de barrios}

Una mirada de algunas problemáticas presentes en las urbes hoy en día, nos muestra la tendencia a la fragmentación social y a la consolidación de procesos de exclusión al interior de las ciudades. Este escenario en un contexto de globalización, pareciera llevarnos a una privatización de la vida de las personas, un repliegue a los espacios más privados, en desmedro del uso de los espacios comunes que nos brinda la ciudad y de las identidades más locales.

En una escala menor, los barrios reflejan estos procesos de fragmentación social y en el caso de algunas urbes, serios problemas de segregación. Los diagnósticos iniciales de los barrios en los que se implementa el Programa, nos han mostrado una gran heterogeneidad de situaciones. Por una parte, barrios caracterizados por bajos estándares de vivienda y espacio público, con déficit de bienes y servicios, con desconexión al resto de la ciudad; y por otra, barrios que más bien sufren procesos de deterioro y degradación al interior de la ciudad. 
No obstante la heterogeneidad encontrada en los 200 barrios, existen algunas problemáticas transversales que tienen que ver con aquellos procesos mayores de exclusión, y que se manifiestan de distintas maneras: individualismo y repliegue en las viviendas, pasividad para enfrentar problemáticas barriales comunes, desconfianza, estigmatización, escaso uso de espacios públicos para la convivencia, uso del tiempo libre dentro del hogar, escasa relación entre vecinos que viven más allá de la propia cuadra, temor y percepción de inseguridad en el espacio público, entre otros.

Como contrapartida de lo anterior, el escenario barrial nos muestra también fortalezas en cuanto al capital social existente en los barrios, historias comunes que delatan una identidad, redes organizativas, apoyo entre las familias del barrio y vecinos que tienden a sentir orgullo por su entorno más inmediato. Todo ello, ayuda a construir una imagen más optimista de recomposición comunitaria, sumado a la percepción de esperanza que los propios vecinos expresan en cuanto a la posibilidad de mejoramiento de sus sectores.

Desde los escenarios urbanos y barriales descritos, el Programa Recuperación de Barrios busca fortalecer las capacidades existentes en éstos para revertir los procesos de deterioro y exclusión, abogando por una mayor equidad en el acceso y uso de los espacios públicos, con políticas inclusivas de la diversidad que fomenten la convivencia ciudadana.

\section{Breve descripción del programa recuperación de barrios}

El Programa Recuperación de Barrios ${ }^{1}$ se orienta al mejoramiento de la calidad de vida de los habitantes de 200 barrios en las quince regiones del país, que presentan problemas de deterioro urbano y vulnerabilidad social, a través de propuestas urbanas integrales basadas en la participación activa de vecinas y vecinos.

Son cuatro los objetivos principales del Programa:

- Recuperar espacios públicos deteriorados;

- Mejorar las condiciones del entorno;

- Fortalecer las relaciones sociales; y

- Propiciar barrios más integrados socialmente

1 Más información del Programa en www.minvu.cl y www.quieromibarrio.cl 
A través del Programa se invierten recursos en la construcción y/o mejoramiento de espacios públicos de los barrios, tales como áreas verdes, equipamientos comunitarios, iluminación y pavimentos, en estrecha relación con un plan social para el uso y apropiación de estos espacios.

El Programa se encuentra estructurado en tres fases que se desarrollan en un período de 2 a 3 años, que tienen por finalidad iniciar un proceso sostenible de recuperación de los barrios.

Durante la primera fase se lleva a cabo un diagnóstico compartido entre vecinos y equipos técnicos, con el que se inicia la construcción del proyecto de recuperación del barrio, el cual se materializa en tres instrumentos: el Plan de Gestión de Obras, el Plan de Gestión Social y el Plan de Gestión de Recursos Complementarios. Como parte de este proceso, se conforma con los vecinos un Consejo Vecinal de Desarrollo (CVD) que al cierre de la fase, suscribe con el municipio y el Ministerio de Vivienda y Urbanismo un Contrato de Barrio, el que contiene aquellos proyectos e iniciativas que han sido priorizados por la comunidad para ser ejecutados en el marco del Programa.

La segunda fase, de más larga duración, es la etapa de gestión y ejecución de las actuaciones comprometidas en el Contrato de Barrio, tanto en materia de obras físicas como de acciones sociales. En esta etapa se considera el fortalecimiento del Consejo Vecinal de Desarrollo en su rol de actor clave de la recuperación y se desarrollan las gestiones de recursos complementarios que permitirán una mayor integralidad en el mejoramiento del barrio. Actualmente, esta es la fase en que se encuentran la mayoría de los barrios en que se implementa el Programa.

Por último, se lleva adelante una fase de cierre, etapa de balance y evaluación del proceso y sus resultados. Esta fase tiene un doble objetivo, en primera instancia, evaluar el avance logrado en materia de recuperación y determinar los desafíos pendientes, y en segundo lugar, construir una Agenda Futura junto al Consejo Vecinal y el Municipio, que proyecte la recuperación del barrio.

Junto a este modelo operativo, se ha definido una estructura de gestión que contempla la participación de un conjunto de actores claves, entre ellos:

- El Consejo Vecinal de Desarrollo (CVD), como espacio de 
representación de los diversos actores barriales, con un rol clave de liderazgo vecinal en el proceso de recuperación.

- Las Secretarías Regionales Ministeriales de Vivienda y Urbanismo, responsables de la ejecución del Programa en cada una de las regiones; y los SERVIU, a cargo de la ejecución e inspección de obras.

- Los municipios, con un rol clave en la gestión del Programa, ya sea como equipo directo en terreno, ejecutor de las obras y/o articulador de los planes de recuperación de barrio dentro del contexto de desarrollo comunal.

- Los equipos de barrio multidisciplinarios, quienes son los que implementan en cada uno de los territorios el Programa, vinculándose directamente con la comunidad y desarrollando las distintas etapas del proceso. Estos pueden corresponder a equipos de municipios, SEREMI de Vivienda y Urbanismo o Consultoras.

- Por último, cabe destacar las mesas técnicas en los distintos niveles, comunal, regional y nacional, todas ellas instancias mixtas de revisión del avance del Programa en los barrios y/o de resolución respecto de los proyectos físicos o definiciones claves del proceso.

\section{Logros de la gestión socio-comunitaria}

A tres años de inicio del Programa Recuperación de Barrios nos encontramos en un momento de balance, el que nos permite compartir algunos de los logros de la gestión socio-comunitaria y recoger los aprendizajes que de la experiencia acumulada se desprenden para una política de recuperación de barrios.

\section{Activación comunitaria: volviendo a verse las caras}

Uno de los primeros logros del Programa ha sido generar espacios para el encuentro vecinal, echar a andar un proceso de animación social a partir del desarrollo de actividades que inciten a los vecinos a salir de sus casas y "volver a verse las caras".

El diagnóstico indica que uno de los principales problemas de los barrios es su repliegue a la vida privada, justamente la falta de una "vida de barrio". Las personas pasan más tiempo al interior de sus 
hogares que usando los espacios públicos, más tiempo atendiendo sus necesidades individuales que las preocupaciones comunes. Claramente no se trata de abandonar los asuntos privados y volcarse plenamente a la vida comunitaria, pero sí de volver a reconocer el valor de la virtud cívica del bien común, del impacto que puede tener en nuestra calidad de vida una mejor convivencia ciudadana y el uso compartido y responsable de nuestros espacios públicos.

En este sentido, los hitos inaugurales, los "puerta a puerta" y las primeras actividades comunitarias que se desarrollan en los barrios, resultan centrales para abrir nuevos espacios donde vecinos y vecinas puedan encontrarse y compartir, comenzando a revertir la tendencia a pasar la mayor cantidad del tiempo libre al interior de los hogares.

"La semana del barrio sirvió para unir a la gente, sobre todo a la alianza mía. Ojalá que se sigan haciendo, en la alianza de nosotros nos vamos a juntar por lo menos una vez al mes para seguir haciendo actividades, o sea folklore, fútbol, así que nos ha servido harto para unirnos como vecinos" (Vecino, Barrio San Hernán, Región de O’Higgins).

Sin duda, la participación no puede quedar reducida solo a "las actividades", pues se busca que los vecinos asuman también un rol protagónicoen la definición yejecución de los planes de mejoramiento barrial. Por ello, el desafío siempre ha sido ir combinando aquellas actividades que aportan a la activación comunitaria y al uso de los espacios públicos con aquellas que permiten generar espacios de deliberación y toma de decisiones sobre el proyecto.

En este sentido, otro de los aspectos clave de la activación de la comunidad ha sido la reactivación de las organizaciones del barrio. El inicio del trabajo siempre presenta el desafío de reconocer los recursos locales, los activos barriales que podrían aportar al proceso de recuperación. Bajo esta perspectiva, la identificación de las distintas organizaciones -formales e informales- existentes en los barrios y la invitación a sumarse al trabajo, es el primer paso para avanzar en una recuperación sostenible.

La diversidad de actividades y tareas que se van desarrollando en el proceso de definición del plan de recuperación, sin duda abren renovados espacios para la participación de las organizaciones, 
generan una mayor visibilización de su trabajo, ayudan a potenciar el intercambio entre ellas y favorecen su legitimidad en la comunidad. Dicho de otra forma, la reactivación de las organizaciones se trata también de un "volver a encontrarse", ya sea entre las propias organizaciones o entre éstas y la comunidad.

En un contexto nacional de baja participación y, en especial, en barrios marcados por una cultura asistencialista, donde la práctica ha sido demandar y recibir más que formar parte de la definición de las soluciones y su implementación, esta activación comunitaria se expresa también en una revaloración de la participación por parte de la comunidad. Los vecinos comienzan a reconocer y valorar las posibilidades que se abren y resultados que se logran al involucrarse más activamente en el proceso, al mismo tiempo que crece el reto del Programa de cumplir las expectativas y abrir espacios efectivos de participación ciudadana.

En suma, creemos que con la llegada del Programa a los barrios se echa a andar un nuevo motor para la vida comunitaria, generando espacios para la sociabilidad y el encuentro asociativo. Es una invitación abierta a participar del proceso de recuperación de los barrios, dando los primeros pasos para transitar desde una noción de beneficiario a la de un ciudadano activo.

“El Programa ya ha aportado, y seguirá aportando a la reactivación de la participación y organización de los vecinos. Ha hecho que vuelvan a interesarse por las personas y el lugar donde viven, que se den cuenta de lo mucho que pueden hacer con los conocimientos y herramientas necesarias" (Vecina, Villa Portales, Región Metropolitana).

\section{Incluyendo a la diversidad de actores}

Un segundo logro de la gestión socio-comunitaria del Programa, tiene que ver con la inclusión permanente en el proceso de la diversidad de vecinos y vecinas de los barrios.

Primero, ello significa entender que una de las características fundamentales de las ciudades es la diversidad de actores que cohabitan en ellas, actores que develan un acceso diferenciado a los bienes y servicios, y particulares valoraciones e intereses sobre el territorio. Segundo, asumir que en una escala menor, esta 
diversidad se manifiesta en los barrios, a través de sujetos sociales con particulares visiones del entorno y sus propias expectativas.

La opción por generar un proceso inclusivo de estos actores y sujetos sociales en un plan de recuperación barrial, ha planteado el desafío de incorporar las distintas miradas que existen entre quienes habitan y transitan cotidianamente en los barrios, a fin de buscar una visión de conjunto, compartida, en definitiva una propuesta de recuperación para todos.

Asumir la diversidad ha supuesto además entender que en los barrios existen diferencias, intereses contrapuestos, brechas culturales y generacionales que se enfrentan tanto en el espacio público como en el privado, generando conflicto en las relaciones vecinales. Con frecuencia estos intereses opuestos tensionan el uso de los espacios, donde un claro ejemplo lo encontramos en la relación entre los jóvenes y los adultos mayores, y de manera más dramática, entre quienes desarrollan actividades ilícitas en el espacio público y aquellos que optan por replegarse en sus viviendas.

Esta diversidad, nos ha llevado a opciones metodológicas con las que se busca incluir a la mayor parte de vecinos y vecinas de los barrios.

En primer lugar, los talleres de autodiagnóstico segmentados por sujetos sociales han sido un aporte interesante en la búsqueda de integrar los puntos de vista de mujeres, hombres, jóvenes, niños, niñas y adultos mayores en la definición de sus principales problemas y en la construcción de una imagen de barrio soñada.

La experiencia del Programa ha mostrado, que a través de este proceso de autodiagnóstico se logran relevar demandas compartidas por todos, como las áreas verdes, los pavimentos, las luminarias; pero también, demandas específicas de ciertos grupos, como los equipamientos deportivos, los jardines infantiles, las sedes comunitarias. Sin embargo, al momento de la priorización de obras, siempre está presente el riesgo de dejar fuera demandas de grupos minoritarios, lo que conlleva un desafío en términos de acuerdos por un proyecto común de recuperación.

En segundo lugar, interesa destacar la apuesta por la conformación de los Consejos Vecinales de Desarrollo como una instancia organizativa 
amplia y plural, lo que ha sido uno de los grandes retos y logros del Programa. La opción por la diversidad de sujetos y actores formales e informales, antiguos dirigentes y nuevos liderazgos ha significado un esfuerzo por incorporar de manera decidida a los vecinos en el proceso de recuperación de sus barrios.

Una primera mirada por los barrios muestra que las organizaciones sociales y territoriales se encuentran en franco proceso de fragmentación y con baja representatividad. Trabajar con cada una de ellas por separado o intentar convocar a otros desde una Junta de Vecinos, desde un Comité Pro-mejoras, o desde cualquier organización funcional existente, lleva aparejada en sí misma la exclusión de todos aquellos actores que no se sienten parte de esa red organizacional. En contrapartida, el Consejo Vecinal de Desarrollo se plantea como una alternativa de integración social, que no reemplaza a las existentes, sino que las convoca a trabajar en conjunto.

Hoy, luego de tres años de Programa, es posible observar el surgimiento de nuevos liderazgos bajo el alero de los Consejos Vecinales de Desarrollo, caracterizados por la diversidad y el interés por trabajar en los espacios comunes y de encuentro entre los vecinos. En general, existe una alta valoración de esta instancia de participación por parte de los vecinos, como espacio para la deliberación y representación de los diversos intereses del barrio.

Quizás uno de los logros a destacar en esta línea es la evidencia de que a través de los Consejos Vecinales se ha logrado incorporar a vecinos que nunca antes habían participado en proyectos colectivos o en otras organizaciones vecinales. Sin embargo, el gran desafío sigue siendo incorporar a los jóvenes al proyecto de recuperación barrial.

La inclusión de la diversidad de actores en los Consejos Vecinales de Desarrollo, se manifiesta en la integración de personas naturales y representantes territoriales que participan asumiendo un rol más activo desde sus pasajes, manzanas, blocks o sectores establecidos en los barrios, representando más del $45 \%$ de los consejeros. Asimismo, los Consejos cuentan con un 54\% de sus integrantes que dan cuenta de la presencia diversa y heterogénea de dirigentes vecinales de organizaciones sociales y territoriales de distinta índole. 
Entre ellas, destaca la participación de las Juntas de Vecinos en la presidencia de los Consejos Vecinales de Desarrollo.

Sin duda, el mayor reto del Programa en esta línea, tiene que ver con el "saldo pedagógico" que se pueda generar a partir del trabajo de los vecinos y dirigentes en un proyecto común desde la diversidad, más allá de la sostenibilidad misma de los Consejos Vecinales de Desarrollo como orgánica funcional.

\section{Construyendo proyectos colectivos}

Un tercer logro de la gestión socio-comunitaria, justamente tiene que ver con la creciente visión de conjunto que se ha ido generando en los barrios. El desarrollo de un espacio común para las organizaciones dentro del Consejo Vecinal de Desarrollo ha permitido ir avanzando en un trabajo colaborativo, al mismo tiempo que la definición de un plan de recuperación para el barrio ha permitido ir articulando el trabajo de las organizaciones en torno a desafíos comunes.

Desde la perspectiva de los Consejos Vecinales de Desarrollo, la presión por los compromisos establecidos con la comunidad en su calidad de representantes y la oportunidad que significan los recursos monetarios disponibles para inversión en obras físicas, impulsan el trabajo conjunto y obligan a ponerse de acuerdo para ir avanzando en la recuperación del barrio y dar respuestas a la comunidad. Sin duda, uno de los desafíos del Programa ha sido ajustar los tiempos de madurez de la organización y su funcionamiento interno, con las demandas de trabajo que tiene prontamente el Consejo Vecinal una vez conformado, favoreciendo un mayor afiatamiento de sus integrantes, el diálogo entre quienes los conforman y la toma de decisiones conjuntas para ir consolidando un proyecto común.

Ahora bien, más allá de la orgánica y funcionamiento de los Consejos Vecinales, que implica ir superando el trabajo fragmentado de las organizaciones y avanzar en una forma de trabajo más colaborativa, se encuentra el tránsito en el barrio desde una visión más individualista hacia la construcción de un proyecto colectivo.

Si las primeras demandas con las que se encontró el Programa eran demandas más bien privadas, asociadas a la esfera del hogar, se ha ido desarrollando una mayor preocupación por lo que sucede de "la reja hacia fuera". Bajo esta perspectiva, lo que se ha ido 
fortaleciendo es el sentido de lo público, una revaloración del barrio en su dimensión social y simbólica, recogiendo la noción de barrio en tanto espacio de las relaciones sociales cotidianas y de las identidades compartidas.

Este tránsito hacia los proyectos comunes ha estado marcado por tres desafíos centrales del Programa:

Primero, la integración de los distintos actores de los barrios en actividades comunes, utilizando el espacio público como escenario del encuentro e intercambio entre vecinos de los distintos sectores, vecinos de distintas edades, género e intereses. Esto es la apuesta permanente por generar espacios de encuentro que vayan fortaleciendo la cohesión social.

"La integración misma de todos los vecinos ha tenido un cambio radical favorable, porque ahora todos estamos unidos, luchando, como se dice vulgarmente, remando para el mismo lado. La cordialidad, el afecto y todas las otras cosas se están viendo para las diversas actividades" (Vecino, integrante del CVD, Barrio Balmaceda, Región de Antofagasta).

Segundo, la definición de un plan de recuperación de barrio que ponga en juego las miradas y sueños de los distintos actores, llegando a elaborar un Contrato de Barrio como resultado de un proceso deliberativo y expresión del consenso sobre una imagen deseada para dicho barrio.

Tercero, la recuperación de la historia de los barrios como actividad central para reconocer las identidades barriales, sus prácticas cotidianas y recursos locales, todos ellos como elementos catalizadores del bien común y plataforma de lanzamiento de una nueva etapa de construcción hacia el futuro de los sectores.

"La iniciativa ha contribuido al fomento de la identidad de nuestro barrio, pues conociendo la historia de nuestros barrios y conociéndonos a nosotros mismos, sabremos dónde llegar" (Vecino, Barrio Leiva, Región del Bío-Bío).

Sin duda, uno de los desafíos del Programa lo constituye la recuperación de lo colectivo, como la base fundamental del proceso de sostenibilidad, donde los actores sociales e institucionales tendrán un rol central. 


\section{Hacia una nueva relación Estado - Ciudadanía}

El Programa Recuperación de Barrios busca iniciar un camino de recuperación que incorpore a los propios vecinos en todo el proceso de definición y desarrollo de un plan integral de mejoramiento de su barrio, lo que supone propiciar una nueva relación entre una política pública y los ciudadanos, donde todos los involucrados comparten niveles de responsabilidad y compromiso en su diseño y ejecución.

Bajo esta perspectiva, uno de los logros del Programa ha sido ir generando una ruptura de la asimetría existente entre los distintos actores involucrados: vecinos, municipio, Ministerio y multisectorialidad, lo que ha venido a instalar nuevas formas de diálogo y trabajo donde todos hemos ido aprendiendo.

En esta relación, aparecen como logros sustantivos, la generación de espacios de deliberación conjunta que se desarrollan durante todo el proceso de la intervención del Programa y que van determinando la toma de decisiones en los barrios.

En términos formales, vale mencionar la conformación y funcionamiento de Mesas Técnicas Comunales, cuya función fundamental es retroalimentar técnicamente el desarrollo del Programa en el barrio, resolver temas clave para la ejecución de los planes de gestión de obras y gestión social, consensuar agendas de trabajo y establecer compromisos de coordinación y producción.

Otra instancia de retroalimentación importante del proceso, son las mediciones de satisfacción que se implementan en dos momentos del proceso, con el fin de incorporar la visión de los vecinos sobre el desarrollo de programa y reorientar las acciones futuras.

Sin duda, un logro fundamental en esta nueva relación Estado Ciudadanía, tiene relación con el avance en la recomposición de las confianzas. Ello, en un escenario barrial en el cual la saturación por intervenciones públicas inconclusas y la desconfianza generalizada en la efectividad de los programas del Estado, aparecían como barreras para la credibilidad del Programa y en definitiva para la participación en el proceso de recuperación.

“Lo que pasa es que hay gente que no creía en esto. Transmitía que 
esto del Programa era un engaño más, que nunca se harían las cosas que nos prometieron. Y ahora que ven que se están mejorando las viviendas, que se está haciendo el Centro Comunitario, recién ahora está confiando, y se acercan para decir que quieren participar, gente que nunca antes había ido a una reunión siquiera" (Vecino, presidente CVD Barrio Ríos Patagónicos, Región de Magallanes).

“... esta es una población de gente de trabajo, y se estaba convirtiendo en un gueto. El programa nos ha sacado de eso y nos ha permitido apropiarnos nuevamente de la población. Es como recuperar el orgullo de vivir aquí" (Vecino, presidente CVD Barrio San Hernán, Región de O’Higgins).

Algunos de los instrumentos fundamentales para este proceso de recuperación de confianza los han constituido la firma del Contrato de Barrio, como símbolo del compromiso de los actores, y la ejecución de las "obras de confianza”, primera muestra tangible de la recuperación del barrio y resultado del trabajo mancomunado del municipio, los vecinos y el Ministerio.

En otro orden, se destaca como central en la generación de confianzas la presencia sistemática y permanente de los equipos de barrio en terreno, incluso con oficinas de barrio funcionales, lo que le da continuidad diaria al trabajo y la certeza que la comunidad necesita para creer y sumarse al proyecto. En la misma línea, el desarrollo de planes comunicacionales que incorporan informativos, asambleas, "puerta a puerta" y diversos tipos de acciones o instrumentos según las particularidades de cada barrio, han resultado centrales para instalar una gestión más transparente y abierta a la comunidad durante todo el proceso.

De alguna manera, la invitación del Programa es a hacer del urbanismo una tarea compartida, superando las planificaciones "desde arriba" e integrando las distintas visiones en una coproducción del mejoramiento barrial.

\section{Proyección de la recuperación barrial: por una mayor autonomía vecinal}

Esta nueva relación Estado - Ciudadanía ha significado también entender el urbanismo como parte del ejercicio ciudadano, asumiendo que la participación de vecinos y vecinas resulta central 
en todo el proceso, desde el diagnóstico hasta la proyección de una agenda futura para los barrios.

Si en la primera fase la participación se centra en las convocatorias amplias para difundir el Programa, definir un plan de recuperación y comenzar a trabajar en conjunto, durante la segunda fase, la participación demanda un mayor involucramiento vecinal en la ejecución y control del plan de recuperación, en miras a la sostenibilidad del proceso.

En esta línea, un aspecto central ha sido el fortalecimiento de los Consejos Vecinales de Desarrollo, abriendo espacios para la toma de decisiones, el desarrollo de sus planes de trabajo y el fomento de su liderazgo en la comunidad. Además, se han ido transfiriendo herramientas de gestión y conocimientos técnicos que fortalecen sus capacidades, por ejemplo en materias de licitaciones, proyectos de obras, planificación, entre otros.

Para ello, ha sido fundamental el trabajo directo y diario entre vecinos y equipos técnicos, compartiendo información de estados de avance y generando un diálogo abierto y claro sobre los distintos aspectos del plan de recuperación del barrio. Sin duda no ha sido fácil, pues muchas veces los equipos de barrio no logran facilitar ni incentivar una participación más activa de los Consejos, dado el apremio del cumplimiento de los plazos y productos asociados a las distintas etapas del Programa.

Ahora bien, junto a los procesos de capacitación, un aspecto central ha sido el aprendizaje "en terreno" que han tenido los Consejos Vecinales, en tanto han ido enfrentándose a los aciertos y dificultades concretas que se presentan al llevar adelante el plan de recuperación de sus barrios.

Dos ejemplos de ello han sido, por un lado, las visitas a las distintas obras en ejecución desde la primera piedra hasta su recepción municipal, y por otro lado, los diseños participativos de algunos proyectos. Ambos han sido centrales para que los vecinos vayan apropiándose poco a poco de sus nuevos o renovados espacios públicos, pero sobre todo para irfavoreciendo una corresponsabilidad en la recuperación del barrio.

Sin duda, este involucramiento activo de los vecinos en las obras no ha estado exento de dificultades, pues el intercambio permanente 
de miradas sobre el diseño y la ejecución de los proyectos ha tensionado los plazos de concreción de las obras y exigido mayores estándares de calidad.

Por su parte, otro avance en una participación más activa e influyente sobre el proceso de recuperación del barrio por parte de los Consejos Vecinales de Desarrollo, ha sido la formación de comisiones de trabajo al interior de éstos o incluso superando el anillo del Consejo. En muchos barrios el Consejo ha liderado la formación de estas comisiones asociadas a los temas que concentran la recuperación del barrio, tales como el medioambiente, la historia e identidad, las obras, la convivencia vecinal, la comunicación. Ello ha permitido que los distintos vecinos puedan sumarse desde las particularidades de sus intereses al proceso de mejoramiento barrial, que se asuman tareas más concretas por parte de la comunidad y responsabilidades claras.

"Cuando me hablaban del CVD yo no entendía mucho ni me interesaba, pero cuando me dijeron que uno podía participar de una comisión de comunicaciones, enganché altiro, porque me encanta eso de las noticias. Me gustó trabajar en el boletín" (Vecina, Barrio José Miguel Carrera, Región del Maule).

Ahora bien, uno de los grandes desafíos para el fortalecimiento organizacional tiene que ver con poder dejar efectivamente capacidades instaladas y liderazgo vecinal, ayudando a garantizar que al concluir el plan de recuperación en el marco del Programa y retirándose el equipo de barrio, los vecinos puedan seguir avanzando en su desarrollo local. Y ello no tiene que ver directamente con que el Consejo Vecinal se mantenga en el tiempo, sino más bien con que existan actores que puedan liderar los procesos comunitarios, llevar adelante una agenda futura, y sobre todo, mantener el sentido de lo colectivo y potenciar la identidad barrial.

\section{Valoración creciente de lo intangible}

Por último, otro aspecto a destacar como logro del Programa, es la relevancia que ha ido adquiriendo en el proceso de recuperación barrial una visión más amplia e integral por parte de los vecinos, relevando la importancia de la activación comunitaria, el encuentro vecinal, la participación y los planes de gestión social como fundamentales en el mejoramiento del espacio barrial. 
Desde esta perspectiva, se ha transitado de una preocupación casi exclusiva por las obras físicas a dimensionar el sentido de las mismas, potenciando el valor social del espacio público y su impacto en la vida cotidiana de quienes habitan el barrio.

“Creo que las obras físicas que se realizarán permitirán que se concreten dentro de la villa muchos espacios sociales. También que los vecinas quieran y cuiden este lugar mucho más, para que los espacios públicos vuelvan a ser hermosos lugares para que los vecinos admiren y disfruten del bello lugar donde viven" (Vecina, Villa Portales, Región Metropolitana).

Asimismo, destaca la importancia que brindan hoy los vecinos, al cambio de imagen de los barrios como uno de los resultados fundamentales del Programa. En esa línea, se valoran aspectos más bien intangibles como la reducción de estigmas asociados a la mirada externa, una percepción de ser mejor vistos por el resto de la ciudad, o una alta valoración de aspectos como embellecimiento del entorno y la importancia de sentirse orgullosos con el barrio.

Un desafío en este ámbito se relaciona con generar compromisos de largo alcance en cuanto al uso, mantención y administración de las obras físicas que den sostenibilidad a la recuperación, fortaleciendo este sentido comunitario que efectivamente comienzan a tener las obras para los propios vecinos.

\section{Conclusiones}

Al cabo de tres años de desarrollo del Programa Recuperación de Barrios, sin duda son muchos los logros y aprendizajes obtenidos en la gestión socio-comunitaria. Trabajar en los barrios ha significado llevar adelante una gestión sectorial más innovadora, donde hemos ido asumiendo que los tiempos de las comunidades no necesariamente son los mismos que los tiempos programáticos, y que se requiere de procesos y espacios adecuados para realmente concretar la participación vecinal que el Programa se propuso como uno de sus ejes centrales.

Más que llegar a los barrios a ejecutar un proyecto ya definido, hemos apostado por acompañar a las comunidades en la elaboración y desarrollo de planes de mejoramiento para sus barrios, reconociendo que son los propios vecinos quienes mejor saben lo que necesitan 
y quienes serán los usuarios más permanentes de los nuevos o renovados espacios públicos.

Sin duda ello no ha sido fácil, ha significado buscar un equilibrio entre la visión técnica y la perspectiva comunitaria, orientando con claridad cuando resulta necesario, pero también sabiendo escuchar y entregando las herramientas para que los propios vecinos vayan tomando crecientemente el liderazgo de las transformaciones de su entorno.

Ha significado también avanzar en la recuperación de los barrios sin precipitar los resultados, asumiendo que la forma de hacer las cosas a veces resulta más transformadora que sus productos, y que para llegar a determinados resultados muchas veces se requiere de procesos de más largo aliento y esfuerzos que realmente le den sostenibilidad a los cambios.

Implementar el Programa nos ha hecho reconocer que ningún barrio es igual a otro, que para desplegar un proceso de recuperación en cada uno de ellos se requieren adaptaciones del modelo y definiciones metodológicas que permitan abordar sus particularidades. No obstante, a pesar de la heterogeneidad barrial, hemos asumido algunos desafíos que son transversales a gran parte de los barrios y sus diagnósticos. Entre ellos, el reto de ir revirtiendo la desconfianza inicial con la que nos encontramos, de ir rompiendo con la desmotivación para participar de manera más activa en el proceso, de suscitar el interés por involucrarse en asuntos colectivos que no necesariamente reportan un claro beneficio individual, de ir desarrollando un trabajo perseverante aunque sus resultados más tangibles se demoren en llegar.

Con estos desafíos, hemos ido avanzado en el desarrollo compartido de propuestas integrales de recuperación de los 200 barrios del país que forman parte del Programa, siendo hoy la sostenibilidad uno de los desafíos más importantes. Ello implica seguir avanzando en la construcción de una ciudadanía más activa, en la apropiación y cuidado de los espacios públicos por parte de los vecinos y vecinas, y en el vínculo del barrio a la ciudad, en especial, a los gobiernos locales y la red multisectorial. 


\section{Referencias bibliográficas}

Aguirre, D.; Aravena, S.; González, A.; Morales, N.; Sandoval, A. (2008). Programa Quiero Mi Barrio: Avances y Desafíos. Temas Sociales, 60, pp. 1-12.

Chávez, O. Y Pons, P. (2008). Satisfacción y participación ciudadana. Un análisis de caso: programa de recuperación de barrios. Informe de práctica de investigación. Manuscrito no publicado. Santiago, Chile: Ministerio de Vivienda y Urbanismo

Chile, Ministerio de Vivienda y Urbanismo (2009). El Diario de las Buenas Ideas. Santiago, Chile: EL MINISTERIO.

(2009). Buenas Prácticas deBarrios. Recuperadoel 2 junio de 2009. De http://www.observatoriourbano.cl

Chile, Ministerio de Vivienda y Urbanismo y Observatorio Social Universidad Alberto Hurtado. (2008). Recuperando barrios de Santiago. Santiago, Chile: AUTOR

Morales, N.; Bustos, C; Aguirre, D. (2009). Encuesta de caracterización familiar y percepción del Programa de Recuperación de Barrios: un análisis de los vecinos, sus comunidades y sus barrios. Cuadernos de Barrio 1, pp. 1-29.

Piña, G.; Torres, M.P. (2008). Mejorando el barrio a través de la participación. Informe de práctica de investigación. Manuscrito no publicado. Santiago, Chile: Ministerio de Vivienda y Urbanismo. 